\title{
Hannsjörg Kowark: \\ Antrittsrede des neuen Direktors anlässlich der Amtseinführung am 22.6.1999
}

Abbildung: Herr Kowark bei seiner Antrittsrede

Sehr geehrter Herr Minister,

Königliche Hoheiten,

Sehr geehrter Herr von Lucius,

Lieber Herr Ehrle,

Liebe Kolleginnen und Kollegen,

Sehr geehrte Damen und Herren,

Für die freundliche Begrüßung und Einführung als neuer Direktor der Württembergischen Landesbibliothek darf ich mich sehr herzlich bei Ihnen bedanken. Ihr zahlreiches Erscheinen anlässlich meiner Amtseinführung zeigt einmal mehr, welchen Stellenwert die Württembergische Landesbibliothek als Kultureinrichtung auf lokaler, regionaler und nationaler Ebene nach wie vor genießt.

Ich freue mich, durch Sie, Herr Minister von Trotha, mit der Leitung einer Bibliothek betraut worden zu sein, die sich in der langjährigen Amtszeit meines Vorgängers, Herrn Dr. Hans-Peter Geh, den ich an dieser Stelle auch ganz herzlich begrüßen darf, weit über die Grenzen Stuttgarts hinaus nationales und internationales Ansehen erworben hat. Man darf ohne Einschränkung sagen, sie hat sich zu einer allerersten Adresse im Kreise der

Landesbibliotheken entwickelt. Ein solches Ansehen ist heutzutage für eine Landesbibliothek keineswegs selbstverständlich. Dieses Ansehen zu erhalten und auf dem jetzt vorhandenen Leistungsstand aufzubauen, ist eine keineswegs leichte Aufgabe, aber eine Herausforderung, die ich gerne angenommen habe.

Mit Dankbarkeit darf ich in diesem Zusammenhang auch die Württembergische Bibliotheksgesellschaft erwähnen, die die Bibliothek stets auf vielfachem Gebiete unterstützt und gefördert hat. Ich freue mich, Herr von Lucius, dass ich auch in Zukunft mit dieser Unterstützung rechnen darf.

Lassen Sie mich deshalb Ihre Worte, Herr Minister, auch als Ermutigung sehen, die Württembergische Landesbibliothek künftig sicher und mit klarem Kurs durch die immer stürmischere See zu steuern. Auf dem zu beschreitenden Weg zeichnen sich allerdings bereits einige Untiefen und auch Klippen ab, die es sicher zu umschiffen gilt.

Mit einem Bestand von knapp 3 Millionen Bänden, ca. 1 Million Entleihungen sowie über 33.000 aktiven Bibliotheksbenutzern zählt die Württembergische Landesbibliothek zu den größten und am stärksten genutzten wissenschaftlichen Bibliotheken Deutschlands. Hinzu kommen die überaus reichen und international angesehenen Altbestände. Ich darf in diesem Zusammenhang nur an die Handschriften-, Inkunabel und Bibelsammlung - eine der bedeutendsten weltweit erinnern. Erst kürzlich ist in unserem Bestand wieder eine sehr seltene und wertvolle Bibel entdeckt worden. Es handelt sich dabei um eine zweisprachige Bibel, die im Auftrag des russischen Zaren Peter des Großen gedruckt worden ist. Zu ihrer Geschichte und Entdeckung darf ich Ihnen unsere aktuelle Ausstellung empfehlen.

Hinzu kommen die Sondersammlungen des Hölderlin und George-Archivs, sowie die überaus bedeutende Musiksammlung.

Nicht zu vergessen ist natürlich auch die Bibliothek für Zeitgeschichte, die mit der

Württembergischen Landesbibliothek traditionell eng verbunden ist.

Die reichen und international einmaligen Bestände der Landesbibliothek, ich darf hier auch auf die Handschriften und Inkunabeln der Fürstlich Fürstenbergischen Hofbibliothek

Donaueschingen hinweisen, sind immer wieder mit sehr interessanten Ausstellungen der Öffentlichkeit präsentiert worden. In den kommenden Jahren wird der Erschließung und Erhaltung dieses kostbaren Altbestandes eine noch viel stärkere Bedeutung zukommen müssen.

Bei einer kritischen Prüfung des augenblicklichen Entwicklungsstandes im Bibliothekswesen ist jedoch unverkennbar, dass wir uns heute in einer der wohl einschneidendsten Umbruchphasen befinden. Aufgaben, Arbeitsweisen und Organisationsstrukturen von Bibliotheken werden sich künftig grundlegend verändern. Nachdem das Leistungsprofil von Bibliotheken über Jahrhunderte hinweg durch die klassischen Tätigkeitsfelder - Sammeln, Erschließen und der Benutzung zugänglich machen - geprägt war, bewegen wir uns im elektronischen Zeitalter mit Riesenschritten auf ein völlig neues Umfeld zu mit virtuellen Bibliotheksbeständen, digitalen Medien und einem für uns Bibliothekare neuen und auch vielfach noch gewöhnungsbedürftigem Benutzerverhalten. Es darf sicherlich als nicht zu weit hergeholt gelten, diese Umbruchphase als zweite bibliothekarische Revolution nach der Erfindung des Buchdruckes zu bezeichnen. War bislang das Buch, das physisch vor Ort gehalten wurde, das wichtigste und fast ausschließliche Medium, das eine Bibliothek definiert hat, so ist heute durch die elektronischen Medien wie CD-ROM, Datenbanken und Internet eine ständig sich verschärfende Konkurrenzsituation zum Buch entstanden. Die elektronischen Medien werden sicherlich das Buch auch auf Dauer nicht verdrängen können, wohl aber zum zweiten Standbein der Informationsversorgung im Bibliothekswesen werden. Dadurch ändern sich die Aufgabenfelder der Bibliotheken wie auch das Benutzerverhalten in drastischer Weise:

- Der Bibliotheksbenutzer erwartet heute im Grunde, dass er jede Art von Information, ob in gedruckter oder elektronischer Form in kürzester Frist geliefert bekommt: am besten an seinen Arbeitsplatz zu Hause, in der Firma oder in der Universität.

- Der Benutzer erwartet, dass die Bibliothek über möglichst lange Öffnungszeiten verfügt.

- Er erwartet, dass die aktuelle und häufig benutzte Literatur frei zugänglich aufgestellt ist.

- Der Benutzer erwartet auch, dass die nicht am Ort verfügbare Literatur in kurzer Zeit zu vertretbaren Kosten von der Bibliothek beschafft werden kann und

- ferner erwartet der Benutzer, dass sämtliche Bibliotheksbestände komplett in einem Online-Katalog nachgewiesen sind und damit rund um die Uhr recherchiert werden können.

Hinzu kommt, dass der Benutzer durch die Vergleichsmöglichkeiten des

Dienstleistungsangebotes einzelner Bibliotheken im Internet kritischer geworden ist. Denn 


\section{durch die Vernetzung der Bibliotheken ist die Monopolstellung der einzelnen Bibliothek
zumindest teilweise verloren gegangen. All dies ist nicht ohne Auswirkungen auf die}

Benutzerstruktur der jeweiligen Bibliothek geblieben. Der Benutzer ist eben nicht mehr für

einzelne Dienstleistungsangebote auf seine bisherige Heimatbibliothek angewiesen, sondern er kann sich für bestimmte Informationsdienstleistungen von seinem Arbeitsplatz aus eine Bibliothek seiner Wahl heraussuchen, die ihm schnelle, effiziente und kostengünstige Informationsmöglichkeiten bietet.

Hier stellt sich natürlich die Frage, wie schnell sich Bibliotheken auf diese neue Herausforderung einstellen werden

Wer Bibliotheken etwas näher kennt, wird sicherlich zugeben müssen, dass sie nicht unbedingt zu den innovationsfreudigsten Einrichtungen zählen. Erfahrungsgemäß reagieren sie meist mit einem mehr oder weniger großen Zeitverzug auf veränderte Rahmenbedingungen. Deshalb gilt für alle Bibliotheken derzeit: wir müssen uns auf neue Publikationsformen, neue Leistungsprofile alle Bibliotheken derzeit: wir müssen uns auf neue Publikationsformen, neue Leistungsprofile
und neue Technologien einstellen.

Die neuen Informations und Kommunikationstechnologien haben dem Benutzer Möglichkeiten an die Hand gegeben, einzelne Bibliotheken völlig zu umgehen, wenn sie seinen Leistungsanforderungen nicht mehr entsprechen und er anderswo seine Informationen schneller, besser und kostengünstiger bekommen kann.

Der Benutzer wird sich deshalb künftig seinen eigenen Informationsmarkt suchen. Für die Bibliotheken bedeutet dies einen verstärkten Leistungsdruck, der zu einer Leistungssteigerung führen muss.

Durch diesen neuen Wettbewerb der Bibliotheken untereinander wird es künftig auch verstärkt zu einer leistungsbezogenen Mittelverteilung der Investitionsmittel durch die staatlichen Träger kommen.

Gleichzeitig eröffnen jedoch die neuen Techniken auch Chancen für neue Dienstleistungen, die Gleichzeitig eröffnen jedoch die neuen Techniken auch Chancen für neue Dienstleistungen, die
es Bibliotheken ermöglichen, völlig neue, nicht orts-gebundene Benutzergruppen zu gewinnen. Ferner können auch die Ressourcen anderer Bibliotheken dem Benutzer am jeweiligen Arbeitsplatz angeboten werden.

All dies bedeutet, dass sich die Bibliotheken derzeit vor enorme Herausforderungen gestellt sehen, die Reformen bzw. eine Änderung traditioneller Strukturen zwingend machen, will man künftig konkurrenzfähig bleiben. Die Zeiten, in denen Bibliotheken nur aufgrund ihrer guten Bestände, nur aufgrund des Pflichtexemplarrechtes und der regionalen Aufgaben unangefochten ihre Position behaupten konnten, sind vorbei. Dies gilt uneingeschränkt auch für die Landesbibliotheken, man denke nur an die Entwicklung in Hessen, wo die Landesbibliotheken derzeit in andere bibliothekarische Systeme integriert werden. Wir haben uns also auf einschneidende Reformen einzustellen, wenn wir uns nicht allein auf eine kulturell traditionelle einschneidende Reformen einzustellen, wenn wir uns nicht allein auf eine kulturell traditionelle
und museale Zielrichtung beschränken wollen. und museale Zielrichtung beschränken wollen.

Vor diesem Hintergrund scheint mir ein Umdenken und eine zielgerichtete Reformpolitik auch in der Württembergischen Landesbibliothek unabdingbar zu sein. Erlauben Sie mir deshalb, auf die vier wichtigsten Eckpunkte künftiger Reformen einzugehen:

\section{Bestandsaufbau}

Neben der traditionellen Pflege des Buchbestandes, der Erschließung von Handschriften und wertvollen Altbeständen sowie der Sondersammlungen gehört es heute zu den Standarddienstleistungen einer modernen Bibliothek, den Bestand an Volltextdatenbanken und elektronischen Auskunftsquellen auszubauen.

Es genügt nicht mehr, sich auf den traditionellen Erwerb von schriftlichen Materialien zu verlassen, sondern die Bibliothek muss bereit sein, für den Benutzer jede Art von Information zu beschaffen und zur Verfügung zu stellen, sei es als Printmedien, als Bild und Tonträger sowie in elektronischer Form

Hier ist zum Beispiel auch an die Sammlung, Erschließung, Pflege und verstärkte Präsentation Hier ist zum Beispiel auch an die Sammlung, Erschließung, Pflege und verstärkte Präsent
von Internet-Quellen für die einzelnen Wissenschaftsfächer zu denken. Ihre Selektion und von Internet-Quellen für die einzelnen Wissenschaftsfacher zu denken. Ihre Selektion und
Präsentation auf den Fachseiten der Bibliothek im Internet ist eine neue Form des virtuellen Präsentation auf den Fachseiten Ier Bibaufbaus innerhalb des Informationsangebotes einer Bibliothek. Auch beim Bestandsaufbau sind Veränderungen in den Grundstrukturen festzustellen. Seit einiger Zeit ist zum Beispiel eine schleichende Verschiebung vom systematischen zum nutzungsorientierten Bestandsaufbau zu beobachten. Dies bedeutet, dass wir unseren Bestandsaufbau, ohne die traditionellen Aufgaben als Pflichtexemplarbibliothek zu vernachlässigen, in Zukunft noch stärker an den Forderungen der Benutzer orientieren müssen.

\section{Bibliotheksetat und Persona}

Die Etatentwicklung der beiden Landesbibliotheken hat seit 1997 nunmehr einen absoluten Tiefstand erreicht, worauf Herr Ehrle bereits ausführlich hingewiesen hat. Die Literaturversorgung gemäß unserem landesbibliothekarischen Auftrag ist derzeit nicht mehr sichergestellt. Unser Bestand weist inzwischen vor allem bei der neueren Literatur empfindliche Lücken auf. Es bleibt mir deshalb an dieser Stelle nur der eindringliche Appell an die Politiker, die eingetretene Stagnation zu beenden. Die vom Land Baden-Württemberg im Rahmen der Zukunftsoffensive Junge Generation zur Verfügung gestellten Mittel, für die wir außerordentlich dankbar sind, tragen sicherlich zu einer gewissen Entlastung der aktuellen Haushaltssituation bei. Dies allein tragen sicherlich zu einer gewissen Entlastung der aktuellen Haushaltssituation bei. Dies allein
reicht jedoch nicht aus, um unseren Aufgaben als Landesbibliothek im Wettbewerb mit anderen reicht jedoch nicht aus, um unseren Aufgaben als Landesbibliothek im Wettbewerb mit andere
Informationsanbietern auch in Zukunft gerecht werden zu können.

Sicherlich sind hier auch die Bibliothekare gefordert, nach zusätzlichen Auswegen zu suchen. Die nicht nur bibliothekarische Tradition, mehr Leistung, neue Aufgaben und Projekte mit der Forderung nach mehr Mitteln oder Personal zu verbinden, hat schon seit längerem keine Relevanz mehr. Dieses Prinzip mag allenfalls in Zeiten des Wachstums gegolten haben. Heute sind neue Strategien, Flexibiliät und Ideen gefragt, um die ständig wachsenden Anforderungen und Probleme auch in Zukunft bewältigen zu können.

Es muss versucht werden, mit weniger Aufwand zumindest gleiche Leistung zu bieten.

Es muss versucht werden, mit weniger Aufwand zumindest gleiche Leistung zu bieten.
Möglicherweise muss sogar eine geringere Qualität in Kauf genommen werden, wenn damit die Möglicherweise muss sogar eine geringere Qualität in Kauf genommen werden, wenn damit d
angebotene Leistung finanzierbar wird.

- Ich denke hier zum Beispiel an längere Öffnungszeiten in den Randzeiten, dann aber mit eingeschränktem Personal- und Dienstleistungsangebot.

- In diesem Zusammenhang denke ich auch an den bereits angesprochenen Nachfrageorientierten Bestandsaufbau sowie

- eine viel engere Kooperation zwischen den großen wissenschaftlichen Bibliotheken in Stuttgart. Dabei meine ich keineswegs eine Fusion der beiden wissenschaftlichen Bibliotheken, die meines Wissens im vergangenen Jahr schon einmal angedacht worden ist. Ich denke hier vor allem an die Erarbeitung von gemeinsamen

Entwicklungslinien und Zielkonzeptionen von Universitäts und Landesbibliothek mit klarer Entwicklungslinien und Zielkonzeptionen von Universitäts und Landesbibliothek
Definition der Aufgabenverteilung. Die heute vorhandenen kommunikations- und Definition der Aufgabenverteilung. Die heute vorhandenen kommunikations-
informationstechnischen Möglichkeiten haben dafür die entsprechenden informationstechnischen Möglich

Zusammenfassend bleibt festzuhalten: personelle und finanzielle Ressourcen lassen sich nicht beliebig kürzen, will eine Bibliothek ihren Leistungsstandard halten oder sogar verbessern. Eine Grundausstattung je nach Auftrag und Benutzerstruktur der einzelnen Bibliothek ist deshalb unverzichtbar. In diesen Zusammenhang gehört auch die Forderung nach einer größeren Flexibilität bei den derzeit noch geltenden haushaltsrechtlichen Vorschriften. Dies gilt vor allem für die beiden Landesbibliotheken. Einnahmen müssen der Bibliothek in voller Höhe und ohne für die beiden Landesbibliotheken. Einnahmen müssen der Bibliothek in voller Höhe und ohne
sonstige Abstriche zur Verfügung stehen. sonstige Abstriche zur Verfügung stehen.

Auch die uneingeschränkte Übertragbarkeit von Haushaltsmitteln muss gegeben sein. Gerade für die Landesbibliotheken gilt, dass man von ihnen erwartet, mehr unternehmerisch tätig zu sein und kostengünstiger zu arbeiten. Allerdings muss auch auf der politischen Ebene darüber Klarheit herrschen, dass Vergleiche mit dem privatwirtschaftlichen Sektor nur dann sinnvoll sind, wenn ein Teil der Hindernisse und bürokratischen Regeln des öffentlichen Dienstes in Zukunft

abgebaut werden können. 
Veränderte Rahmenbedingungen erfordern jedoch auch auf der bibliothekarischen Seite mehr als
bislang ein strukturelles Umdenken

bislang ein strukturelles Umdenken. Ideen- und Erfindungsreichtum sind deshalb mehr denn je gefragt.

\section{Benutzung}

Die Schlagworte Kundenorientierung und Dienstleistung sind für viele Bibliothekare mit Sicherheit kein Neuland mehr, leider klingen sie für manchen Bibliothekar immer noch fast revolutionär.

Eine ausgeprägte Serviceorientierung muss deshalb heute als selbstverständlich gelten und ist im Grunde inzwischen auch zu einer Existenzfrage für Bibliotheken geworden.

Meines Erachtens müssen die Nutzerbedürfnisse permanent evaluiert werden. Dies bedeutet aber auch, dass das Benutzungs und Informations-verhalten der Benutzer in viel stärkerem $\mathrm{Maße}$ als bisher beobachtet und analysiert werden muss, bevor die Bibliothek ihre Leistungsziele definiert.

Kein Industriebetrieb würde heute Produkte auf den Markt bringen ohne vorher Angebot und Nachfrage sorgfältig untersucht zu haben.

Jede Mitarbeiterin und jeder Mitarbeiter ist deshalb aufgefordert, dazu beizutragen, bei jedem Nutzerkontakt die Nutzerbedürfnisse herauszufinden und zu überlegen, was zu einer Verbesserung des Angebotes der Bibliothek beitragen könnte.

Die Wünsche der Benutzer sollten für die Bibliothek Grundlage seine, ihre Ziele zu definieren. Ich hoffe, dass die Zeiten, in denen Bibliothekare immer schon wussten, was der Benutzer will, endgültig vorbei sind.

Benutzerforschung als erweiterte Marktforschung wird in Zukunft eine noch viel größere Rolle in den Bibliotheken spielen müssen.

Es darf als unstrittig gelten, dass eine Reihe von Standarddienstleistungen in jeder modernen Bibliothek als Leistungsparameter vorhanden sein sollte. Ich darf hier nur einige der wichtigsten einmal aufzählen:

- Aufbau eines Online-Kataloges, der den Gesamtbestand der Bibliothek umfasst.

- Zügige Bestellung der zur Anschaffung vorgesehenen Literatur mit sofortigem Nachweis im elektronischen Katalog der Bibliothek.

- Große Freihandbestände für die aktuelle und häufig ausgeliehene Literatur. Reine Magazinbibliotheken wie die Württembergische Landesbibliothek sind diesbezüglich stark benachteiligt. Hier kann in Zukunft nur durch einen Erweiterungsbau Abhilfe geschaffen werden.

- Angebot elektronischer Dokumentlieferdienste. Hier treten die Bibliotheken zunehmend in Wettbewerb zu anderen Bibliotheken bzw. zu sonstigen Anbietern, so dass dieser Dienstleistungssektor ständig überprüft und erweitert werden muss.

- An letzter Stelle sind großzügige Öffnungszeiten zu nennen. Traditionelle Schließzeiten und verkürzte Öffnungszeiten sollten nach Möglichkeit der Vergangenheit angehören. Denn geschlossene Bibliotheken sind totes Kapital, das man sich in heutiger Zeit nicht mehr leisten kann.

\section{Organisation}

Die bisher genannten Anforderungen an eine moderne und leistungsorientierte Bibliothek setzt eine Definition von entsprechenden Leistungszielen voraus. Nicht die Fortführung von Traditionen wird künftig eine sichere Basis für Erfolge sein können, sondern die aktuelle Leistungsfähigkeit. Hierzu wird es absolut notwendig sein, zu prüfen, ob wirklich schon alle

Rationalisierungsreserven ausgeschöpft sind. Ohnehin bietet uns die jetzt eingeleitete Umstellung auf ein integriertes Bibliothekssystem die einmalige Chance, die gesamte Organisationsstruktur den veränderten Verhältnissen anzupassen.

Jede Bibliothek wird in Zukunft zu beweisen haben, dass sie auf dem Markt bibliothekarischer Leistungen in Abhängigkeit von Angebot und Nachfrage eine erfolgreiche Position einnehmen kann.

In den Bereich der veränderten Arbeitsorganisation gehört auch der Aufbau von kooperativen Bibliotheksstrukturen. Dies gilt insbesondere für die drei großen wissenschaftlichen Bibliotheken in Stuttgart. Hier ist mit dem Bibliotheksinformationssystem der Region Stuttgart und den bereits definierten Zielsetzungen eine solide Basis geschaffen worden.

Um all diese Anforderungen umsetzen zu können, müssen vor allem die Mitarbeiterinnen und Mitarbeiter der Bibliothek entsprechend vorbereitet und geschult werden. Voraussetzung hierfür ist ein Grundverständnis für die modernen Informationstechnologien mit all ihren Chancen und Risiken. Der Bibliothekar benötigt heute eine profunde Kenntnis der Informationswege und der weltweit angebotenen Informationsquellen. Entscheidend wird dabei immer sein, die Übersicht über den Informationsmarkt zu behalten.

Für den Bibliothekar werden in Zukunft kommunikative Fähigkeiten noch in viel höherem Maße zu den Grunderfordernissen seines Berufes zählen. Letztlich bedingt der technologische Umbruch im Bibliothekswesen eine neue Art der Fortbildung, die sich auf die Bibliothek als Ganzes bezieht. Kontinuierliches Lernen und Innovationsbereitschaft sind aus diesem Grunde unverzichtbar.

Ziel der kommenden Jahre muss es sein, die Württembergische Landesbibliothek im Sinne des skizzierten Leistungsprofils unter Beibehaltung und Weiterentwicklung ihrer traditionellen Aufgaben zu einem Informationszentrum unter Anwendung modernster Kommunikationstechnologien zu machen.

Schließen möchte ich mit einem Wort des Dankes an meine Mitarbeiterinnen und Mitarbeiter: Sie haben mich hier mit Freundlichkeit und Offenheit empfangen und mir damit die ersten Schritte in diesem Hause leicht gemacht.

Einen Namen darf ich in diesem Zusammenhang ganz besonders hervorheben:

Sie, liebe Frau Schneider, haben bereits die Stellvertretung von Herrn Geh wahrgenommen und waren dann fast zwei Jahre kommissarische Direktorin dieses Hauses. Für die Fairness, die Sie mir vom ersten Tage an entgegengebracht haben und für die Sachkompetenz, mit der Sie mich in diese Bibliothek eingeführt haben, möchte ich Ihnen aufrichtig danken.

Ich bin mir sicher, dass wir beide ein sehr gutes Team bilden und bestens zusammenarbeiten werden. Bei diesen Voraussetzungen bin ich guter Hoffnung, dass es mir gelingen möge, meine Kolleginnen und Kollegen in der Württembergischen Landesbibliothek für die künftigen Aufgaben zu begeistern. 